\title{
Piloted Simulation Assessment of the Impact of Flexible Structures on Handling Qualities of Generic Supersonic Aircraft
}

\author{
Mary T. Stringer ${ }^{1}$, Brandon Cowen ${ }^{2}$, Keith D. Hoffler ${ }^{3}$ and Jesse C. Couch ${ }^{4}$ \\ Adaptive Aerospace Group, Inc., Hampton, VA, 23666 \\ and \\ Marilyn E. Ogburn ${ }^{5}$ and Corey G. Diebler ${ }^{6}$ \\ NASA Langley Research Center, Hampton, VA, 23681
}

\begin{abstract}
The NASA Langley Research Center Cockpit Motion Facility (CMF) was used to conduct a piloted simulation assessment of the impact of flexible structures on flying qualities. The CMF was used because of its relatively high bandwidth, six degree-of-freedom motion capability. Previous studies assessed and attempted to mitigate the effects of multiple dynamic aeroservoelastic modes (DASE). Those results indicated problems existed, but the specific cause and effect was difficult to ascertain. The goal of this study was to identify specific DASE frequencies, damping ratios, and gains that cause degradation in handling qualities. A generic aircraft simulation was developed and designed to have Cooper-Harper Level 1 handling qualities when flown without DASE models. A test matrix of thirty-six DASE modes was implemented. The modes had frequencies ranging from 1 to $3.5 \mathrm{~Hz}$ and were applied to each axis independently. Each mode consisted of a single axis, frequency, damping, and gain, and was evaluated individually by six subject pilots with test pilot backgrounds. Analysis completed to date suggests that a number of the DASE models evaluated degrade the handling qualities of this class of aircraft to an uncontrollable condition. Further conclusions will be provided when analysis is complete.
\end{abstract}

\section{Nomenclature}

$\begin{array}{ll}C I R & =\text { Control Influence Rating } \\ C M F & =\text { NASA Langley Research Center Cockpit Motion Facility for flight simulation } \\ D A S E & =\text { Dynamic Aeroservoelastic (referring to modes) } \\ G F D & =\text { Generic Flight Deck (cab mounted on the CMF's base) } \\ H S C T & =\text { High Speed Civil Transport (Boeing Ref. H aircraft evaluated during HSR) } \\ H S R & =\text { High Speed Research program at NASA Langley in the 1990s } \\ R Q R & =\text { Ride Quality Rating } \\ S P A R C 6 & =\text { Scalable Prototyping Aircraft for Rapidly Configured } \underline{6} \text { DOF model }\end{array}$

\footnotetext{
${ }^{1}$ Research and Development Engineer, 100 Exploration Way, Suite 330, Hampton VA 23666, AIAA Student Member.

${ }^{2}$ Formerly of Adaptive Aerospace Group, 100 Exploration Way, Suite 330, Hampton VA 23666, AIAA Member.

${ }^{3}$ President, Senior Research and Development Engineer, 100 Exploration Way, Suite 330, Hampton VA 23666, AIAA Associate Fellow.

${ }_{5}^{4}$ Engineer- Intern, 100 Exploration Way, Suite 330, Hampton VA 23666, AIAA Student Member.

5 Aerospace Engineer, D-317 Flight Dynamics Branch, 8 Langley Blvd. MS308, Hampton VA 23681 and AIAA Associate Fellow.

${ }^{6}$ Aerospace Engineer, D-317 Flight Dynamics Branch, 8 Langley Blvd. MS308, Hampton VA 23681.
} 


\section{Introduction}

$\mathrm{T}$ HE effects of dynamic aeroservoelastic (DASE) structural modes on pilot performance and aircraft handling qualities has been a topic of interest for decades. While it has been a problem on various aircraft over the years, a recent driving factor for this interest lies with the fact that supersonic civilian transport aircraft concept designs are becoming larger, more slender and as a result, more flexible. In the late 1990s, NASA's High Speed Research program (HSR) implemented a DASE model on a motion simulator and found significant handling qualities issues. The team developed 20 different DASE parameter variations from the baseline dynamics with varying structural mode application, structural stiffness (implemented with frequency gains), modal damping, control effector excitation levels, DASE visual cues and turbulence levels. ${ }^{1}$ The goal was to track which of these potential triggers and solutions were identifiably major contributors to the adverse effects or potential mitigators. During the study, it became obvious that in addition to the pilot induced oscillation tendencies, there was also noticeable biodynamic coupling. Biodynamic coupling refers to the incidents when structural dynamics couple through a pilot's arm to the control inceptor to sustain the motion and sometimes results in divergent motion. While these findings certainly provided great insight, they failed to point to which particular DASE models were objectionable by way of the mode characteristics. Isolating this piece of information for use in future design purposes was the main goal of this study. The subject work was sponsored under the NASA Fundamental Aeronautics Program, High Speed Project as the results and implications are significant to their ongoing projects related to sonic boom reduction and supersonic civil transport.

A generic airplane simulation model known as the Scalable Prototyping Aircraft for Rapidly Configured 6DoF (SPARC6) was developed for the simulation study. SPARC6 parameters were set to give the aircraft performance similar to that of the High Speed Civil Transport (HSCT) Cycle-4. ${ }^{2}$ Aircraft dynamics were set to provide CooperHarper $^{3}$ Level 1 or 2 handling qualities for a transport-category aircraft landing-task. Various DASE models were added to the baseline or nominal aircraft. In the earlier study, DASE models from the full aircraft design were used. These models had three modes at low enough frequencies to impact the pilot in each axis making it difficult to identify with any certainty which mode was the main driver of bio-dynamic coupling and which axis was the most significant in terms of handling quality degradation. For the current study, the team opted to separate both DASE modes and axes for independent evaluations. The result was a test matrix that included the baseline aircraft with no DASE and a matrix of configurations, each with a single DASE frequency and damping on either the symmetric (longitudinal) or anti-symmetric (lateral) axis.

Six pilots from the Department of Defense, NASA and industry participated as test subjects, and all but one flew the entire test matrix. They provided oral feedback as well as three scaled-rating assessments. The results from these evaluations are presented in this paper. Conclusions, including insight on the effects of DASE on handling qualities, ride quality, and suggestions for follow-on work to better understand the phenomenon, are made after the data analysis is presented. Potential mitigation techniques will be addressed in future publications.

\section{Simulation Modeling and Testing}

The original HSCT model was available for use, but its highly detailed implementation made it very difficult to employ modifications that were well understood. There was too much potential for multiple effects of any change. There was also a desire to make results more generalized and broadly applicable. The research team matched the general performance of the HSCT and tuned the handling qualities parameters to provide Level 1 Cooper-Harper Ratings (CHR) in several areas. The Level 1 or 2 parameters were based on transport-category aircraft landing-task specifications from MIL-STD-1797A. ${ }^{4}$ To maintain the high desired level of generality and have direct control of model elements, the SPARC6 simulation model was selected.

\section{A. SPARC6 Simulation Setup}

The generic model concept is not a new one. Hoffler, et al. ${ }^{5}$ developed the basis for the SPARC6 model during the Generic Fighter Model research at NASA Langley in the 1990s. This general fighter model was useful to the research at hand because the full 6 degree-of-freedom nonlinear equations of motion along with numeric integration were already included in a single block that is fed calculated forces and moments for the current state. This concept of a compartmentalized simulation tool was modified to describe the system specifics of a generic supersonic transport platform. Ref. 6 is a detailed report on the structure and utility of this simulation model which provides figures that show the connection of the block components discussed. The rigid body model was developed based on work previously done by Hoffler, et al ${ }^{5}$ and the characteristics of the HSR Ref. H Cycle-4 configuration ${ }^{2}$.

A DASE model with a matrix of independent symmetric and anti-symmetric frequencies, damping, and gain was added to SPARC6 for this study. The resulting generic aircraft model had rigid body and flexible structural 
components. The flexible structure model was excited by rigid body loads and directly impacted cockpit motion but did not affect aircraft aerodynamics. The result was that the DASE models could only impact aircraft trajectory through the pilot's inputs. The resulting generic model in its supersonic transport configuration developed for this work has performance similar to the HSR Ref. H Cycle-4, Cooper-Harper Level 1 or 2 handling qualities, and a matrix of single mode/single axis DASE models. The DASE augmented SPARC6 model was implemented in NASA Langley's Cockpit Motion Facility and utilized the Generic Flight Deck.

Inside the SPARC6 model, the stick inputs pass through parabolic stick shaping, dead band, filters and gains to command pitch rate in the longitudinal axis and stability axis roll rate in the lateral axis. The simulation was designed for feet-on-the-floor flying meaning that the rudder pedals are not used. This was acceptable for the current study because no crosswind was simulated.

The stick inputs were then passed to the pre-determined linear models for dynamic response matching with respect to angular rates. These linear models represent second order short period dynamics in the longitudinal axis, whereas a first order roll mode defines the roll and yaw response in the lateral axis. The stick commands proportional roll and yaw rates for a coordinated, stability axis roll, and sideslip and sideslip feedback were used to eliminate gravitational effects and other sideslip drift. The damping and lead terms for the longitudinal axis were scheduled with angle of attack. The desired rates from the linear model were compared to the rates calculated from the current 6DOF nonlinear system's actual state. Subsequently, gains were used to require certain forces and moments from the system to follow the linear model for the next cycle. Tables of trimmed lift, drag, and side force were utilized to compute longitudinal, normal, and side forces. A table lookup engine model with a first order lag supplied the thrust values. Essentially, this linear model formed the desired rigid body dynamic response of the nominal aircraft in simulation. A simple first order lag function was used to model the actuators. The required accelerations were passed through limiting functions to provide control power limitations as a means to approximate loss of control scenarios. It should be noted that aircraft departure (loss of control) was modeled, but post departure was not. Additionally, no aerodynamic loss of control scenarios were experienced in this study.

The SPARC6 simulation was developed in MATLAB ${ }^{\circledR} /$ Simulink $^{\circledR}$ and the associated auto-code generator was used to implement the models in NASA Langley's Cockpit Motion Facility (CMF) simulator for piloted

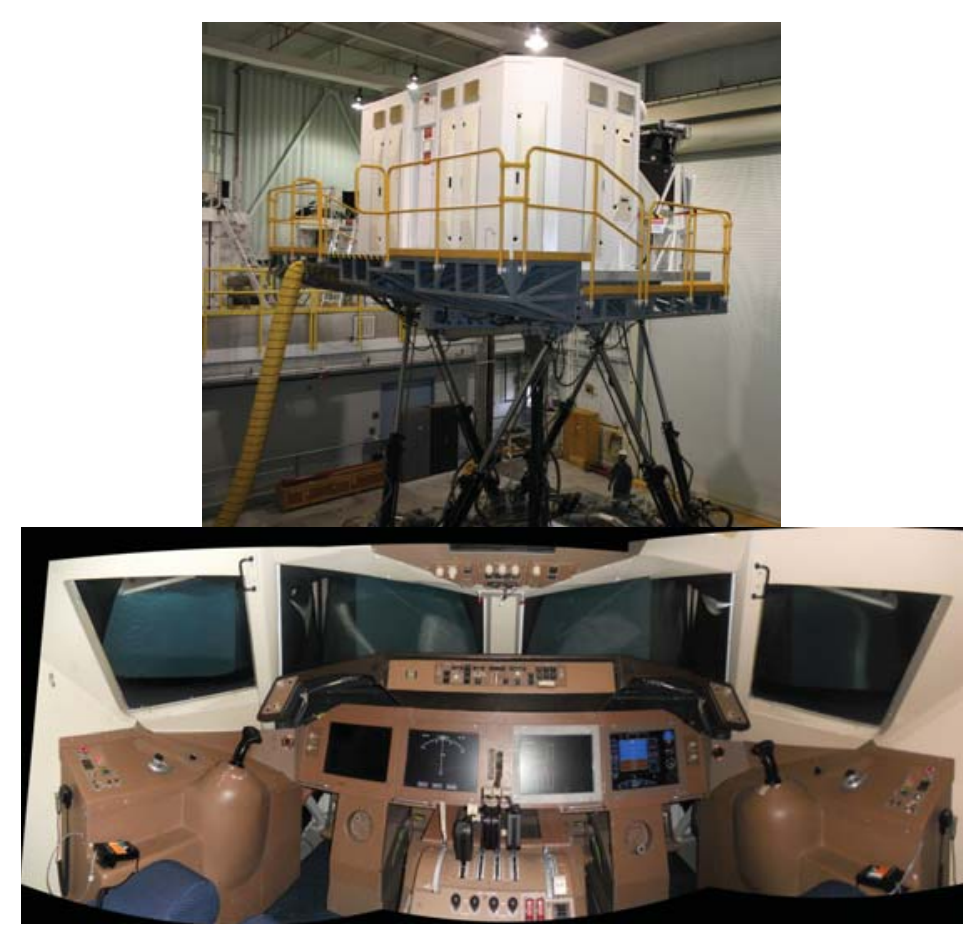

Figure 1. NASA Langley CMF (top), GFD cockpit interior (bottom).

evaluations. The CMF is a $6 \mathrm{DOF}$ hexapod motion simulator with a $3 \mathrm{~Hz}$ production control law. For the purpose of this simulation, the Generic Flight Deck (GFD) was the cab mounted on the motion base. Fig. 1 depicts the interior and exterior of the CMF/GFD that was used in this study. This full motion simulator is the successor to the Visual Motion Simulator (VMS) used in the HSR studies in the 1990s. This simulator is still in its first year of research use and the operational limits and latencies are yet to be fully quantified. 

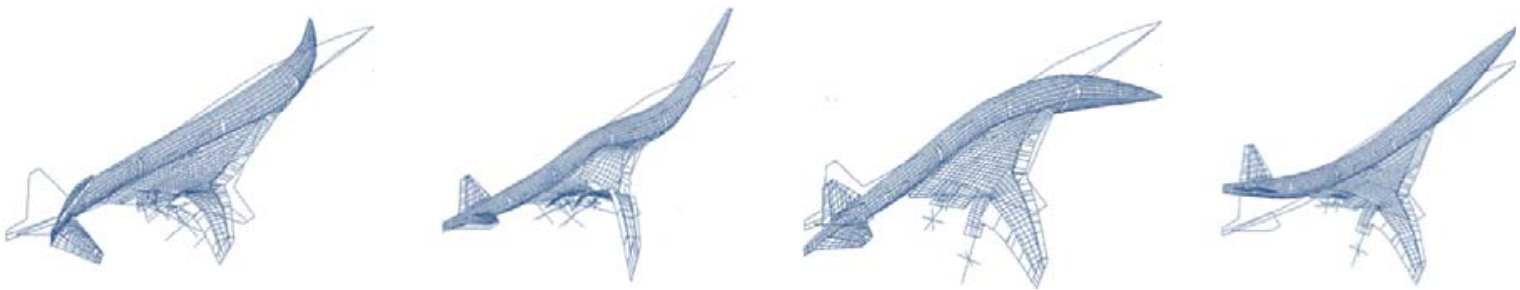

Figure 2. Various flexible mode shapes.

\section{Cooper-Harper Handling Qualities Rating Scale}

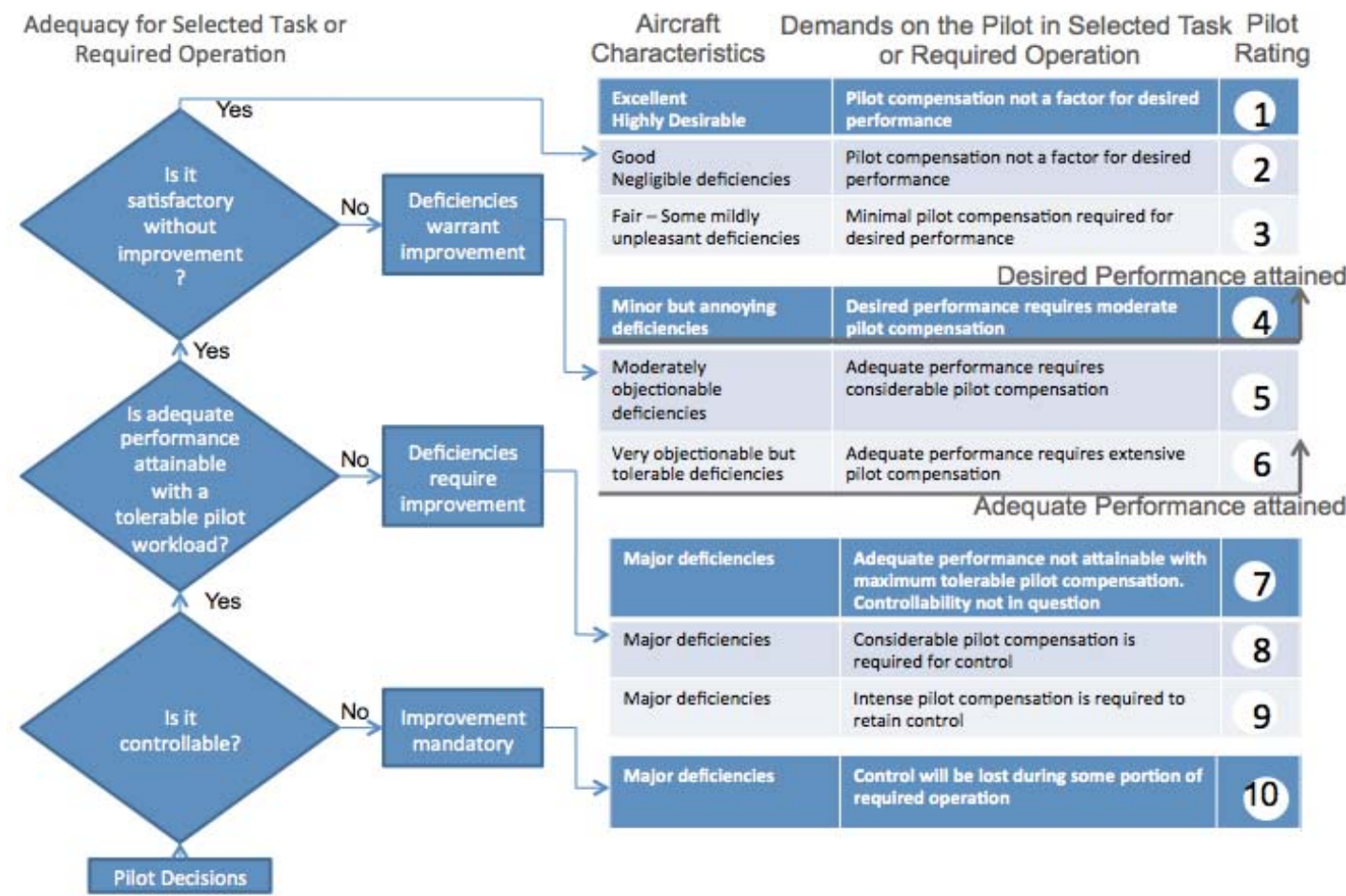

\section{DASE Influence on Ride Quality}

Cockpit vibrations do not impact ride quality.

Cockpit vibrations are perceptible but not objectionable: no improvement necessary.

Cockpit vibrations are mildly objectionable: improvement desired.

Cockpit vibrations are moderately objectionable: improvement warranted.

Cockpit vibrations are highly objectionable: improvement required.

Cockpit vibrations cause abandonment of task: improvement required.

\section{Dynamic Aeroservoelastic (DASE)} influence on pilot's control inputs

CIR

Pilot does not alter control inputs as a result of aircraft flexibility.

Pilot intentionally modifies control inputs to avoid excitation of flexible modes.

Cockpit vibrations impact precision of voluntary control inputs.

Cockpit vibrations cause occasional involuntary control inputs.

Cockpit vibrations cause frequent involuntary control inputs.

Cockpit vibrations cause sustained involuntary contro inputs or loss of control.

Figure 3. Piloted simulation rating scales. 


\section{B. Dynamic Aeroservoelastic Models (DASE)}

For the purpose of this simulation study, each structural mode is modeled as an individual second order function that determines translational deflection at the pilot station. The deflections are driven by the torque, and therefore indirectly by the resulting force applied at the control surface aerodynamic center in the respective axis. Single bending modes are used during the piloted study to isolate the impact of specific frequencies and damping on pilot control strategy. Mode frequency and amplitude are used to compute accelerations at the pilot station due to these fundamental flexural modes of vibration. The forcing functions that drive the flexible structure were derived from the control surface loads which have been determined in order to generate the angular accelerations of the rigid body simulation as described above. Fig. 2 shows the general deformations (exaggerated for clarity) that a flexible supersonic transport aircraft could exhibit. ${ }^{1}$ It is these individual bending modes that the DASE models simulate. For the purpose of the piloted-simulation study, thirty-six DASE models were included in the test matrix. They represent two axes - symmetric and anti-symmetric, six frequencies per axis $-1 \mathrm{~Hz}$ through $3.5 \mathrm{~Hz}$, and three deflection ratios per axis - ranging from nominal to five times the nominal deflection ratio. Given the simulator's stated $3 \mathrm{~Hz}$ production control law capability, the data generated from the $3.5 \mathrm{~Hz}$ DASE models has been examined to determine if the excessive phase lag associated with operating at a higher frequency has corrupted the data. It should be noted that these DASE modes are activated only by control effector excitation, and not external sources such as turbulence- which is different from the HSR study. Turbulence was not modeled during any of the production runs associated with this work.

\section{Piloted Simulation Detail}

Six subject pilots participated in the study. Five pilots were active research pilots, while the other, though retired, had extensive experience with this type of aircraft. As a group, they represented NASA (2), industry (3), and the military (1). Their experience ranged from business jets and large transports to military bombers including the B-1B. Prior to data collection, the research team modified the Hyperlinked-Overview of Piloted Evaluations (HOPE) ${ }^{7}$ database for storing and searching data for piloted studies so that the data and comments collected during the study would be well-documented and easily accessed and sorted. The handling qualities were rated by each pilot based on the Cooper-Harper Rating Scale, as well as a DASE influence on Ride Quality Rating (RQR) scale and a DASE influence on pilot's control input - Control Influence Rating (CIR) scale. The RQR and CIR scales were developed and used during the HSR study presented in Ref. 1. These scales are shown in Fig. 3. The pilots were allowed a number of practice runs during the first half-day session to familiarize themselves with the nominal, rigid body aircraft. Then, they were introduced to all 36 DASE models in a random order, which varied by pilot. The pilots were not informed as to which DASE model, if any, they were flying. The task, or mission, was two part. The first phase was an Instrument Landing System (ILS) capture and tracking task in instrument meteorological conditions (IMC). The pilots were asked to rate this portion separately to gauge DASE impact on precision control. There were also scorecard items for this phase in order to provide incentive to be on the ILS. The ILS itself was usually offset from the runway centerline by $300 \mathrm{ft}$, requiring a large lateral correction when the pilots broke out of IMC and could see the runway. The first segment of the second phase covered the lateral correction to the runway centerline starting when the aircraft broke out of the clouds at an altitude of approximately $250 \mathrm{ft}$. above the threshold. The final segment then began at an altitude of $50 \mathrm{ft}$. and included the flare and touchdown portions of the task. The lateral correction required an aggressive lateral maneuver due to the low altitude at which the correction should have been initiated. The pilots were briefed that this maneuver was going to most likely be more aggressive than they would normally attempt and there was no option to wave off. This large lateral maneuver was implemented to increase the pilots' directional activity and accordingly the chances of exciting the DASE modes. The ILS lateral offset was randomized each run to appear on either the left or right of (or sometimes on) the runway centerline to reduce the pilot's ability to anticipate the runway location. The straight-in runs were included to reduce predictability and occurred around one out of every 10 runs. When straight-in runs showed up the run was repeated so each rated set nominally included three offset landing tasks. Fig. 4 is a graphical depiction of the two part task. 


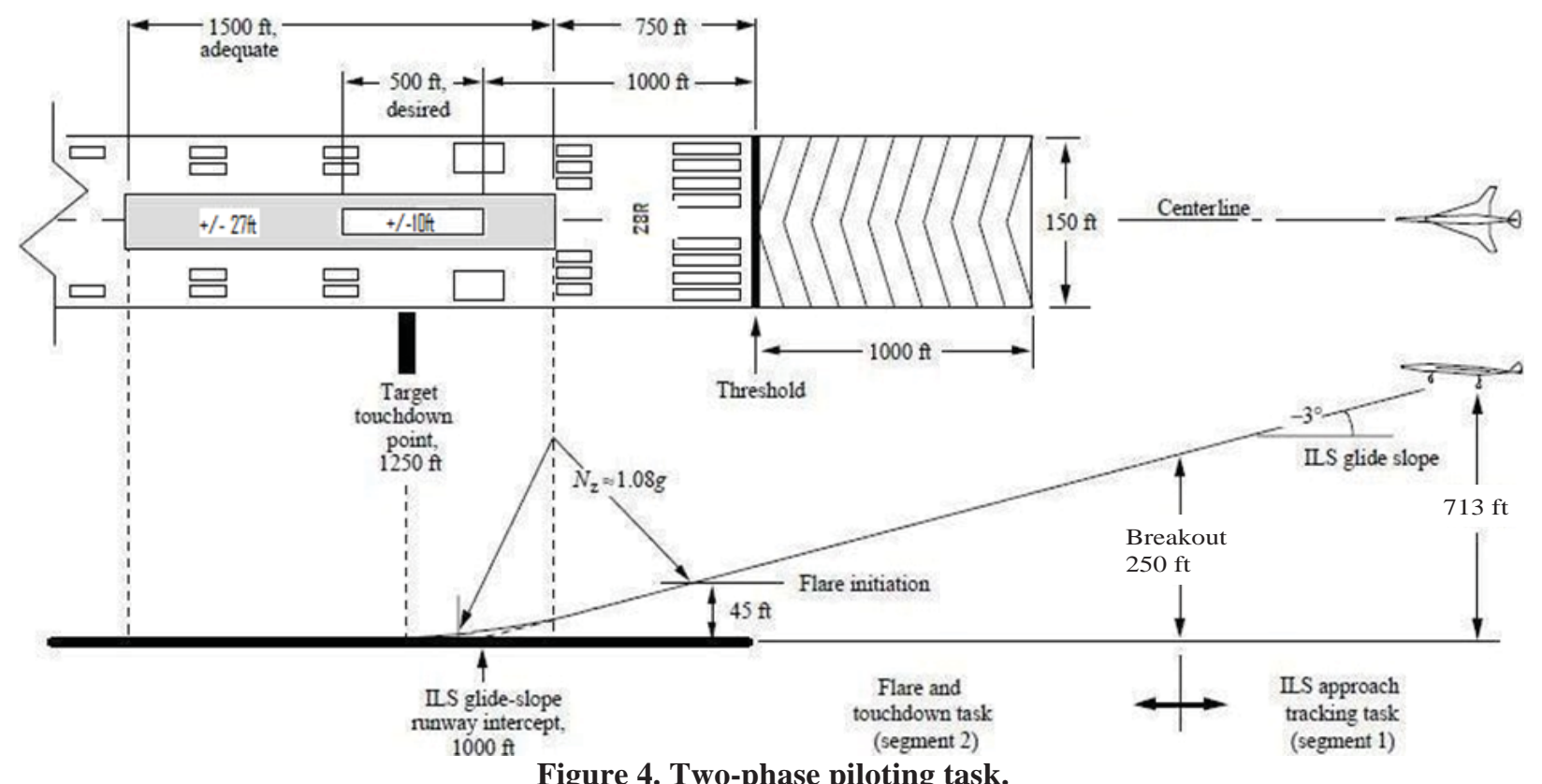

Table 1 shows the target, desired and adequate grading metrics for the two-phase task. Three runs were given for each model unless the pilot felt ready to assess the model after an earlier run. This was often the case for the more objectionable DASE models.

\begin{tabular}{|l|c|c|c|c|}
\hline \multicolumn{1}{|c|}{ Performance Metric } & Parameter Name & Target & Desired & Adequate \\
\hline Airspeed deviation at 50 ft (kts) & AS_50ft_Err & 0 & $+/-5$ & $+/-10$ \\
\hline Maximum bank angle less than 50 ft $\left(^{\circ}\right)$ & Ban__It_50ft_Err & 0 & $+/-5$ & $+/-7$ \\
\hline X distance of m.g. from runway threshold (ft) & X_MG_TD_Err & 1250 & $+/-250$ & $-500,+1000$ \\
\hline Y distance of $\mathrm{m.g}$. from runway centerline (ft) & Y_MG_TD_Err & 0 & $+/-10$ & $+/-27$ \\
\hline Landing hdot $(\mathrm{ft} / \mathrm{s})$ & Ldg_Hdot & 0 & $>=-4$ & $>=-7$ \\
\hline Landing heading error $\left(^{\circ}\right.$ ) & Ldg_Hdg_Err & 0 & $+/-3$ & $+/-6$ \\
\hline $\begin{array}{l}\text { Localizer deviation descending from 325 ft } \\
\text { through 275 ft msl }\end{array}$ & LocDev_300ft & 0 & $1 / 2$ diamond & 1 diamond \\
\hline $\begin{array}{l}\text { Glideslope deviation descending from 325 ft } \\
\text { through 275 ft msl }\end{array}$ & GSDev_300ft & 0 & $1 / 2$ diamond & 1 diamond
\end{tabular}

Table 1. Pilot performance metrics.

\section{Results and Analysis}

The piloted-simulation study was concluded in the fall of 2012. Over 130 data variables were collected for every run. The pilots were offered the opportunity to comment after each simulation run, but after each model's three run series, they were required to make comments, evaluate the vehicle's handling qualities, and go through the scorecard rating. There were at total of 36 DASE models and one no-DASE model used in this study. The scorecard ratings were documented and the pilot comments were transcribed from the recordings. There are approximately 850 nonpractice runs that were recorded and are available for analysis. Of those runs, there were a number that did not go through touchdown. The reason for this could have been mechanical system aborts or pilot/test engineer/simulator technician aborts. In certain DASE vehicle/pilot scenarios, the commanded accelerations and resulting simulator leg position commands either exceeded or appeared to be about to exceed a position-error threshold/ hydraulic accumulator pressure deficit for the system, which prompted an abort. These runs were not included in the 3-run series for evaluation unless the pilot felt that he was able to rate and provide comments on the particular DASE vehicle based on the events of the aborted run and any other runs that he performed. In only three instances were 
ratings given by a pilot to a vehicle that was not flown to a landing at least once, meaning that the mission was not flown to completion by that pilot. These three ratings were all CHR 10 for the landing task and have been included in the analysis.

\section{A. Ratings Mapping}

The three-dimensional graphs in Fig. 5-8 are a visual summary of the handling quality impacts due to the various frequencies and deflection ratios. These graphs provide separate views of the different axes with regards to their DASE model ratings impact. On the x-axis is the range of tested DASE frequencies. On the y-axis are the deflection ratio multipliers, and on the z-axis is the average rating given to the vehicle. For each plot, a point representing the "No DASE" vehicle average rating has been placed at the $(0,0,-)$ point to indicate that it was a 0 amplitude, 0 frequency model. This serves as a good benchmark for the remainder of the plotted data. Note that for all four rating scales, the No DASE vehicle consistently received the best average rating or comparable rating.

The rating averages were calculated on a by-series basis, noting that there are cases where a particular pilot might have seen the vehicle more than once or another pilot did not see the vehicle at all. For example, Pilot 4 did not experience or rate 5 of the vehicles due to scheduling issues. The instances in which an individual pilot flew a particular vehicle twice are a result of an effort to later analyze data trends with regards to a 'learning curve'. Time permitting, the pilot would unknowingly be given a repeat vehicle series that he flew early in his sequence. The average ratings shown in Figs. 5-8 included these second series runs as this information is valuable to the overall vehicle assessment. After the $\mathrm{x}-\mathrm{y}-\mathrm{z}$ matrix was assembled and plotted using MATLAB function 'surf', the command 'interp shading' was utilized to interpolate linearly throughout the matrix and shade the plot accordingly ${ }^{8}$. The color bar to the right of each plot allows for the three-dimensional plot to be properly read from all angles, so as to not misrepresent the height of the average rating. Note that the color representations change as the rating scales change from Cooper Harper 1-10 scale, and the other 1-6 scale.

A quick scroll through the ratings plots shows the effect that was anticipated by the study team. Generally speaking, the low frequency DASE vehicles received the worst ratings. At the higher frequencies, 3-3.5Hz, the deflection ratio became less of a factor in the assigned rating. This was more prevalent in the anti-symmetric axis. The distinction made between the axes held true throughout most of the ratings- the deflection ratio had a stronger impact on the pilot's vehicle preference in the symmetric axis.

\section{Ride Quality Rating}

The DASE influence on ride quality average ratings shows large variation over the 1-3.5Hz range. Fig. 5 contains the corresponding plots. In the symmetric axis, the slope is mostly consistent over the deflection ratio. However, the maximum deflection ratio tested was able to drive the higher frequency vehicles into the RQR 2-3 range which indicates that the vibrations were perceptible and sometimes mildly objectionable. While the vehicle average ratings peaked at around 5.5 in both axes, six vehicles (five symmetric axes and one anti-symmetric axis) received pilot ratings of 6 which indicates that the vibrations caused abandonment of the task. By this, the pilot was indicating that he was no longer attempting to perform well with regards to the flight technical scorecard, but was only trying to safely land the aircraft and in some cases felt that this was no longer achievable as well. These six vehicles all were $1-1.5 \mathrm{~Hz}$ frequency. It is worth pointing out that of these six vehicles, two received RQR ratings of 3 by other pilots. In the $3-3.5 \mathrm{~Hz}$ range in the anti-symmetric axis, the deflection ratio appears to have caused no change in rating, but below that frequency range, the impact was substantial. 

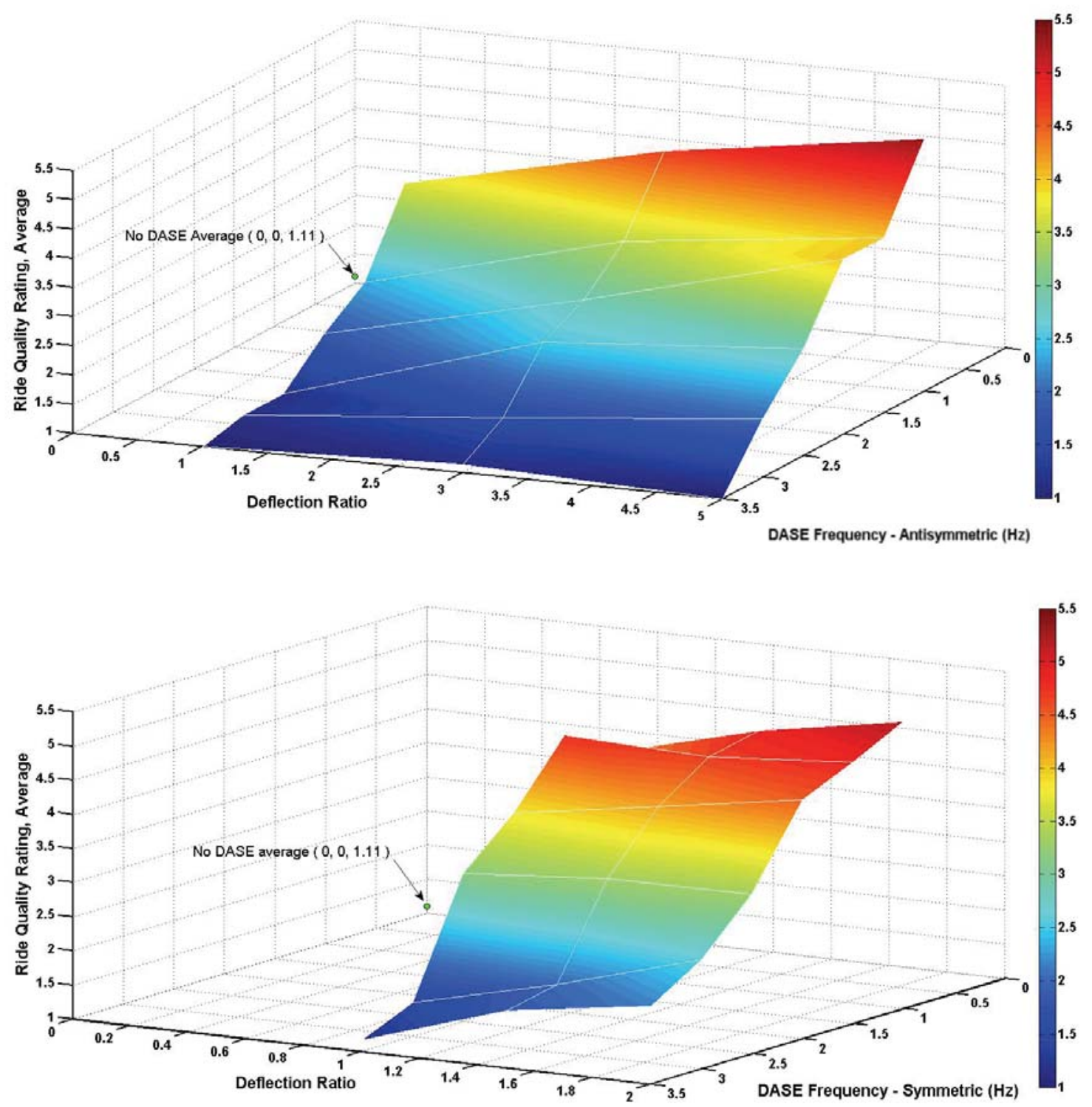

Figure 5. Ride Quality Ratings summary, average by vehicle, plotted by axis: anti-symmetric DASE vehicles (top), symmetric DASE vehicles (bottom).

\section{Control Influence Rating}

The DASE influence on the pilot's control inputs was primarily a function of the DASE frequency, for both DASE axes. Fig. 6 contains the corresponding plots. In the anti-symmetric axis, there is little variation in the average rating for the 2.5-3.5 Hz range from the No DASE vehicle. The No DASE vehicle average rating was 1.04. This rigid body aircraft would be expected to receive a consistent rating of 1, indicating that the pilot did not alter his control inputs as a result of the aircraft flexibility. However, it should be noted that the pilots were not informed as to the vehicle that they were flying and could have perceivably thought they felt some type of vibration that would have warranted changing their gain to reduce the chance of exciting the seeming DASE model. Six vehicles received a maximum rating of 5 or 6 from at least one pilot. Four of those six were 1Hz DASE model vehicles (two in each DASE axis). This level of CIR rating indicates that the pilot experienced frequent or sustained involuntary control inputs and recognized that the vibrations due to exciting an elastic mode were not only impacting his control precision but also causing involuntary inputs. These involuntary inputs are referred to as biodynamic coupling. The initial analysis of this phenomenon will be discussed in a later subsection. 

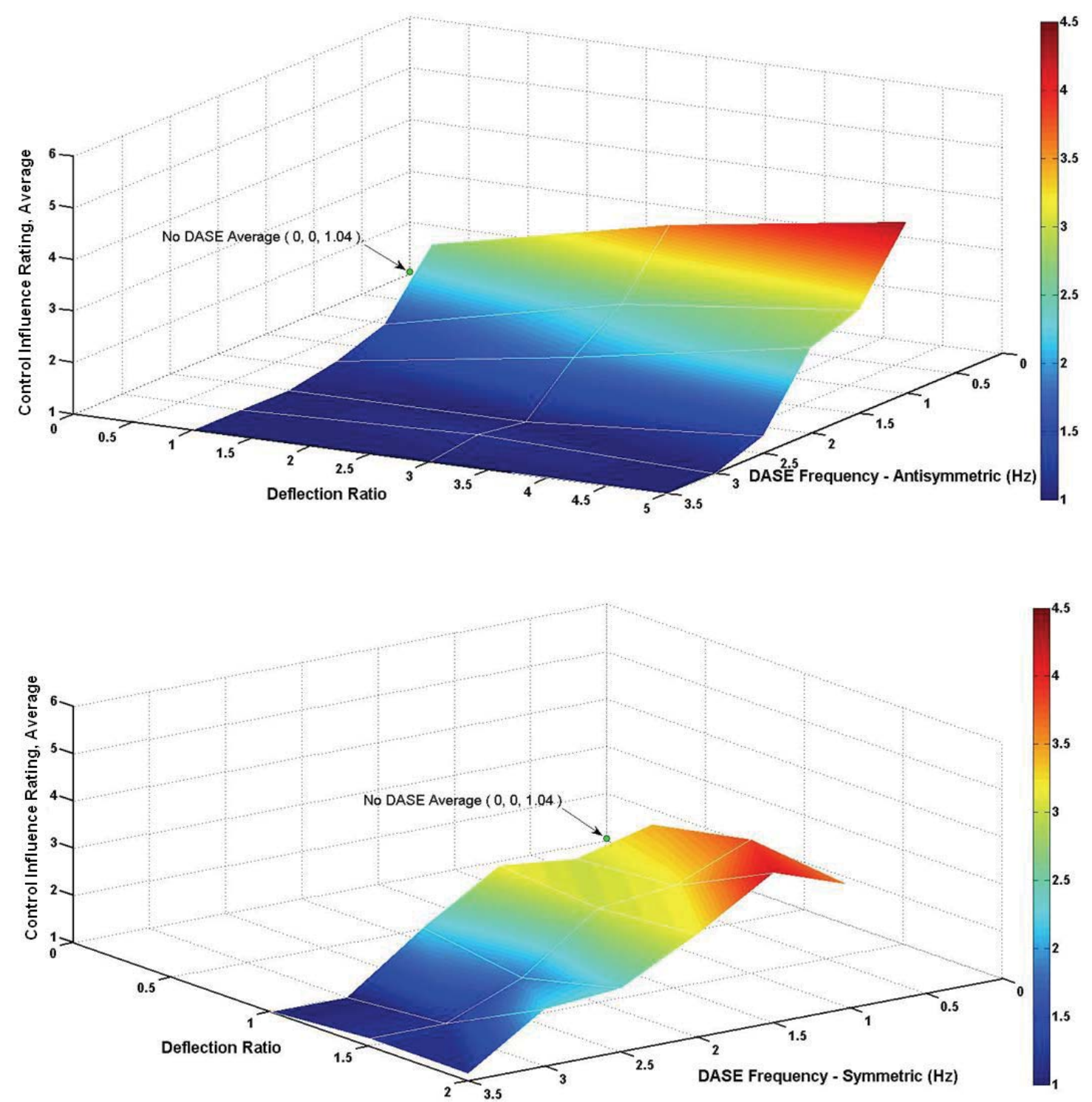

Figure 6. Control influence Ratings summary, average by vehicle, plotted by axis: anti-symmetric DASE vehicles (top), symmetric DASE vehicles (bottom).

\section{ILS Tracking- Cooper Harper Rating}

The Cooper Harper ratings assigned to the ILS tracking task are found in Fig. 7. The slope for frequency to rating becomes drastically steeper starting at about $2 \mathrm{~Hz}$ - this is more pronounced in the anti-symmetric axis. The plots illustrate that the average ratings were worse for the symmetric than the anti-symmetric models for all frequencies except the lowest one, $1 \mathrm{~Hz}$. One of the 37 vehicles received a maximum rating of 10 by one or more pilots. There were no ratings of 9 assigned. However, there were 6 separate vehicles that had a maximum rating to minimum rating span of 4 or 5 . This is a good indicator that the ILS handling qualities were very pilot dependent and a correlation to control strategy can most like be made at the completion of all analysis. 

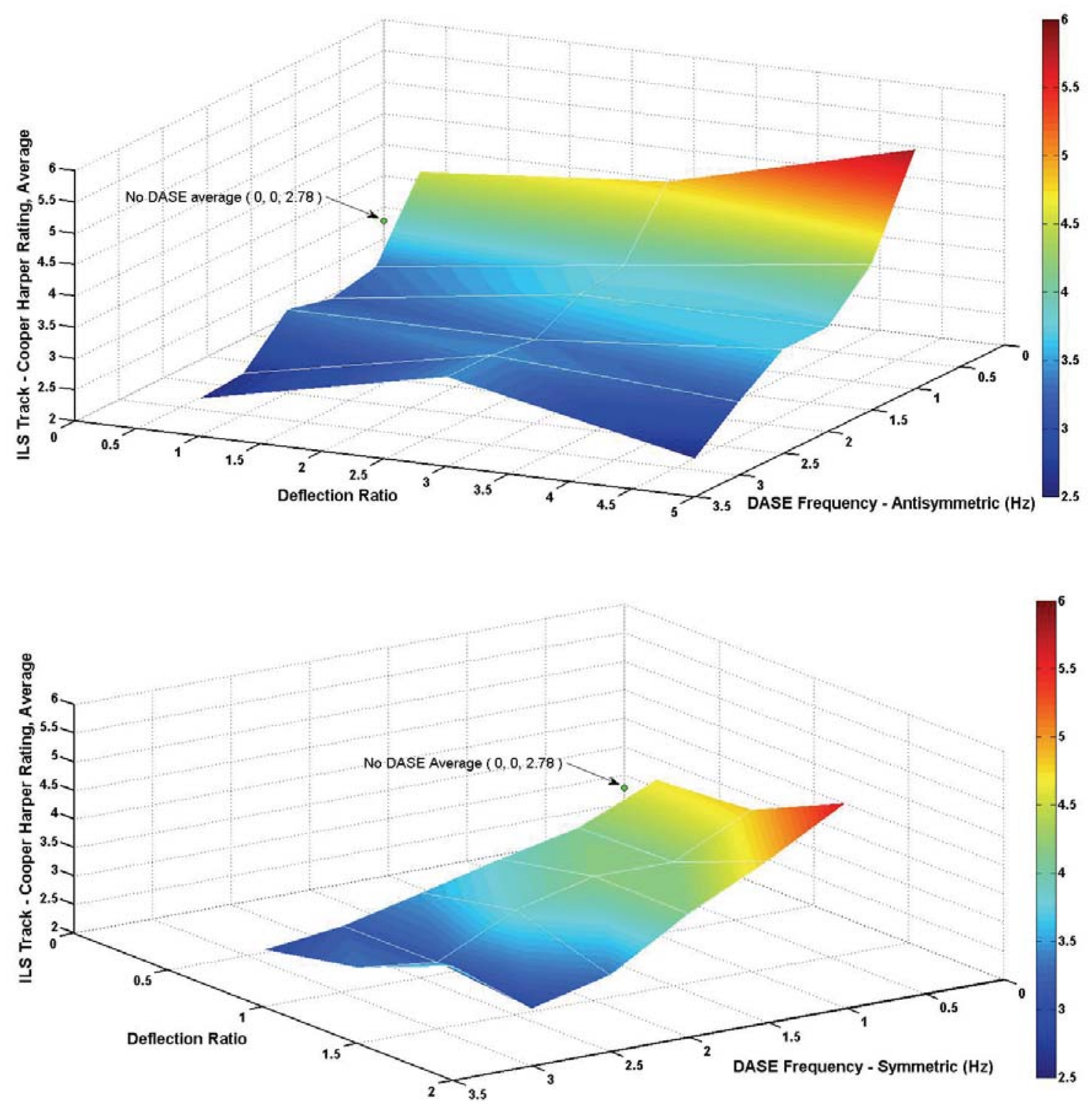

Figure 7. ILS Tracking- Cooper Harper Ratings summary, average by vehicle, plotted by axis: antisymmetric DASE vehicles (top), symmetric DASE vehicles (bottom).

\section{Landing- Cooper Harper Rating}

Overall, the Cooper Harper ratings for the landing task were consistently higher than for the ILS tracking task in both axes, as well as for the No DASE rigid body vehicle. This indicates that the handling qualities of the particular vehicle were more impactful on the precision of the flying during the landing task. This could easily point to the statement that the symmetric axis is the more sensitive axis regarding DASE modal frequencies. This is verified by the fact that the band of green, yellow and red takes up a much larger portion of the frequency axis in the symmetric axis plot of the LCHR ratings. For the most part, from the range of $2.5-3.5 \mathrm{~Hz}$ in the anti-symmetric axis, the ratings stayed very close to those of the No DASE vehicle. The maximum ratings of 10 were given to six vehicles, five of which were symmetric models. For some of these six vehicles, the best rating given by any single pilot was as good as 4. This is definitely due in part to the fact that the 'higher gain' pilots were more likely to excite the modes in some cases. Four separate vehicles had a maximum-to-minimum rating span of 5 to 6 . Fig. 8 contains the corresponding plots. 

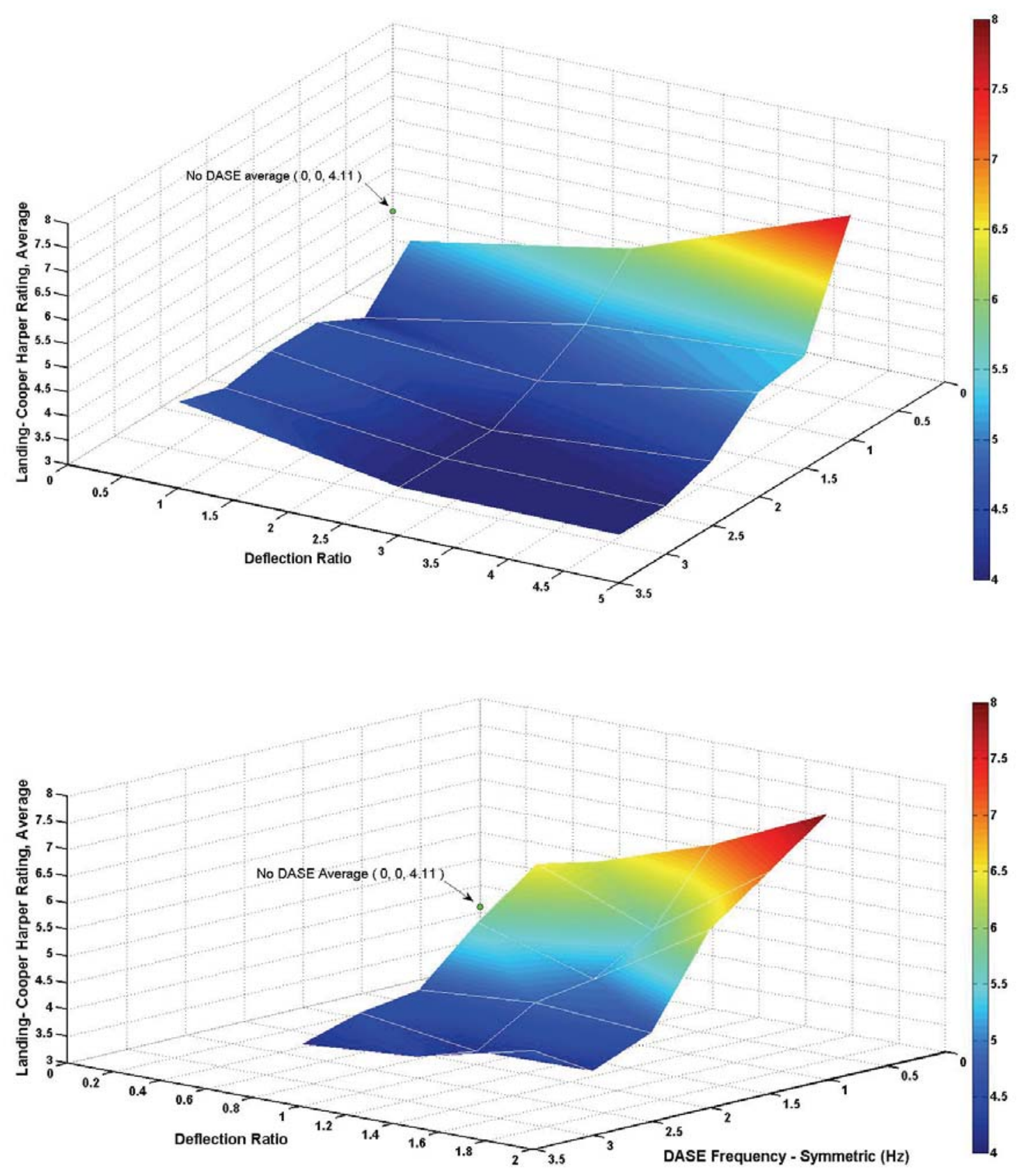

Figure 8. Landing- Cooper Harper Ratings summary, average by vehicle, plotted by axis: anti-symmetric DASE vehicles (top), symmetric DASE vehicles (bottom). 


\section{B. Control Strategy}

Early analysis of the control strategy employed by the various pilots yielded a basic distinction between the pilots. For all three runs on each vehicle, the pilot's maximum stick deflection, average stick deflection, maximum angular rate, and mean angular rate were averaged to generate a by-vehicle average. After this was completed, the 37 vehicles were then averaged to produce the numbers in Table 2. This method made certain that vehicles without three runs for particular pilots were not deemphasized, and that all vehicles received equal weighting, meaning that no vehicle which required larger or smaller inputs to maintain control or avoid activation of a DASE mode was emphasized. The general information that comes out of this analysis is the typical stick deflections for each pilot. It can easily be seen that Pilots 1 and 5 were generally speaking "higher gain" pilots than $2,3,4$, and 6 . It is also interesting to correlate the higher-gain piloting approach to overall flight technical error as seen in Table 3: pilots 1 and $5 \mathrm{had}$ on average the best performance of the group. This average was done on a by-run basis. The reason for this is mainly due to the fact that the vehicle seemed to have very little impact on the flight technical error. While the errors clearly varied by run, and the maximums ran the gamut, there was no pattern or correlation that has been identified to the vehicle.

\begin{tabular}{|c|c|c|c|c|}
\hline \multicolumn{5}{|c|}{ Pitch Input Summary - Averaged Over Vehicles } \\
\hline PILOT & Max Rate & Max Stick & Mean Rate & Mean Stick \\
\hline 5 & 2.25 & 0.71 & 0.21 & 0.08 \\
\hline 1 & 2.58 & 0.80 & 0.22 & 0.07 \\
\hline All & 2.26 & 0.64 & 0.16 & 0.05 \\
\hline 6 & 2.79 & 0.80 & 0.16 & 0.05 \\
\hline 4 & 2.26 & 0.58 & 0.13 & 0.04 \\
\hline 3 & 1.99 & 0.53 & 0.11 & 0.03 \\
\hline 2 & 1.75 & 0.43 & 0.10 & 0.03 \\
\hline
\end{tabular}

\begin{tabular}{|c|c|c|c|c|}
\hline \multicolumn{5}{|c|}{ Roll Input Summary- Averaged Over Vehicles } \\
\hline PILOT & Max Rate & Max Stick & Mean Rate & Mean Stick \\
\hline 1 & 8.55 & 1.63 & 0.86 & 0.18 \\
\hline 5 & 8.95 & 1.59 & 0.69 & 0.14 \\
\hline 6 & 8.49 & 1.62 & 0.49 & 0.11 \\
\hline All & 7.88 & 1.42 & 0.51 & 0.10 \\
\hline 3 & 7.70 & 1.44 & 0.39 & 0.09 \\
\hline 4 & 7.99 & 1.30 & 0.42 & 0.07 \\
\hline 2 & 5.78 & 0.95 & 0.22 & 0.05 \\
\hline
\end{tabular}

Table 2. Pilot input summary for maximum and mean peak to peak stick deflection and rate by axis: pitch (left), roll (right).

\begin{tabular}{|c|c|c|c|c|c|c|c|c|}
\hline PILOT & AS_50ft_Err & Bank_It_50ft_Err & LocDev_300ft_Err & GSDev_300ft_Err & Ldg_Hdg_Err & Ldg_Hdo_Err & X_MG_TD_Err & Y_MG_TD_Err \\
\hline 5 & 0.976 & 4.435 & 0.178 & 0.250 & 0.680 & 4.806 & 201.187 & 5.971 \\
\hline 1 & 0.774 & 4.791 & 0.166 & 0.181 & 0.710 & 4.438 & 248.035 & 5.996 \\
\hline 2 & 1.043 & 3.663 & 0.423 & 0.344 & 1.972 & 3.784 & 293.207 & 12.680 \\
\hline All & 1.021 & 4.189 & 0.318 & 0.295 & 1.126 & 4.289 & 332.630 & 8.541 \\
\hline 6 & 1.080 & 4.120 & 0.500 & 0.467 & 1.087 & 4.459 & 384.269 & 7.261 \\
\hline 4 & 1.007 & 3.908 & 0.270 & 0.237 & 1.253 & 3.865 & 387.796 & 7.702 \\
\hline 3 & 1.250 & 3.951 & 0.335 & 0.277 & 1.045 & 4.314 & 471.722 & 11.538 \\
\hline
\end{tabular}

Table 3. Pilot summary for average flight technical error listed by increasing longitudinal distance between the aircraft c.g. and the touchdown point. (See Table 1 for definition of parameter names)

\section{Pilot Induced Oscillations and Biodynamic Coupling}

Pilot induced oscillations (PIOs) have been defined by researchers and industry in a variety of ways and to varying extents throughout the years. A quick summary of the key features of each of these definitions follow. PIOs occur when the response state (the pitch attitude or roll attitude most typically) is approximately 180 degrees out of phase with the pilot's intended input. This input is considered to be intentional on the part of the pilot to control the aircraft, but instead results in an unintentional sustained or uncontrollable oscillation. The amplitude of the oscillation can be constant, divergent or even convergent and there is no minimum number of cycles required for classification. Many definitions indicate that a requirement for a true PIO is that the oscillations must become 'intrusive' on the pilot's task. A severe PIO is considered to be any oscillation that meets the above requirements and causes the pilot to abandon the task in order to focus all efforts on maintaining control of the aircraft. There are references in the literature indicating that a PIO with less than 10 degrees per second of peak-to-peak angular rate is considered 'mild', but clearly this is very vehicle dependent'.

In the early 2000s, Hoh Aeronautics, Inc. received both United States Air Force and NASA funding to develop a tool that would identify Pilot Induced Oscillations in real-time, for both test flights as well as piloted simulation studies. The result of this effort is a tool named ROVER (Realtime Oscillation VERifier). The delivered software was verified to accurately detect PIOs in the first half-cycle oscillation $89 \%$ of the time when well-tuned to a particular aircraft or simulator. 
The method employed by the ROVER software is a four-point check. It first assumes that all aircraft response is an oscillation. Because aircraft attitude is not easily monitored and is a non-zero mean signal, ROVER chooses to monitor rate signals vice attitude signals. As the derivative of position, rate lags position by 90 degrees. This means that with regards to phase, a PIO will be the pilot's input at 90 degrees out of phase with the rate. It searches through the output data, in the case of a PIO this is the aircraft rate, and marks all local maximums and minimums as triggers. It then calculates the frequency between neighboring half cycle peaks using the time delta. The phase difference between that particular rate peak and the corresponding input's neighboring oscillation is calculated. Once this is completed, the peak-to-peak magnitudes of both the input and output oscillations are determined. This identification process via the ROVER algorithm was used to process the $850+$ runs from the current study. The flagging routine was rewritten and repurposed to meet the thresholds appropriate for the vehicles, specifics of the DASE modes, as well as the inclusion of a search for biodynamic coupling. The output of ROVER with the adjusted thresholds for peak-to-peak amplitudes can be seen in Table 4. Note that a score of '4' was received when a half-cycle oscillation met all four of the flag requirements: frequency within designated band, phase shift within acceptable band for PIO, peak-to-peak stick input amplitude above tuned threshold, and peak-to-peak rate amplitude above tuned threshold. The re-written post-processing algorithm used in this study required the frequency and phase requirements to be met before a flag could be set for amplitude. The reason for this being that without those two requirements, the oscillation was of no interest to the study. Based on the final two thresholds, the level number of severe PIO instances identified will vary. Relatively small stick input is often an indicator of fairly common pilotaircraft coupling scenarios such as "pitch bobble" and "roll ratchet", or an unintended input cause by biodynamic coupling for instance. Thus, the proper tuning to the individual vehicle is essential for accurate results.

Pitch PIO ROVER Output Summary
\begin{tabular}{|c|c|c|c|c|c|c|} 
Vehicle & AS Freq & S Freq & Defl Ratio & All Pilots & High Gain Pilot & Low Gain Pilot \\
4 's & 4's \\
\hline 113 & 0 & 1 & 1 & 13.44 & 20.13 & 3.67 \\
\hline 107 & 0 & 1 & 1.5 & 9.33 & 14.67 & 3.33 \\
\hline 101 & 0 & 1 & 2 & 7.71 & 11.50 & 5.00 \\
\hline 102 & 0 & 1.5 & 2 & 6.84 & 16.33 & 2.67 \\
\hline 103 & 0 & 2 & 2 & 6.75 & 17.75 & 1.00 \\
\hline 104 & 0 & 2.5 & 2 & 6.42 & 13.50 & 2.25 \\
\hline 114 & 0 & 1.5 & 1 & 6.12 & 11.57 & 0.67 \\
\hline 108 & 0 & 1.5 & 1.5 & 5.48 & 10.00 & 2.25 \\
\hline 127 & 2 & 0 & 3 & 5.22 & 5.00 & 3.75 \\
\hline 106 & 0 & 3.5 & 2 & 5.00 & 10.67 & 2.67 \\
\hline 115 & 0 & 2 & 1 & 4.94 & 7.33 & 3.67 \\
\hline
\end{tabular}

Roll PIO ROVER Output Summary
\begin{tabular}{|c|c|c|c|c|c|c|}
\cline { 7 - 7 } Vehicle & AS Freq & S Freq & Defl Ratio & All Pilots & High Gain Pilot & Low Gain Pilot \\
4 's & 4's \\
\hline 113 & 0 & 1 & 1 & 9.04 & 15.63 & 1.67 \\
\hline 114 & 0 & 1.5 & 1 & 8.88 & 18.14 & 0.00 \\
\hline 115 & 0 & 2 & 1 & 8.39 & 12.33 & 3.00 \\
\hline 119 & 1 & 0 & 5 & 8.32 & 13.50 & 2.00 \\
\hline 112 & 0 & 3.5 & 1.5 & 8.22 & 15.67 & 1.33 \\
\hline 132 & 1.5 & 0 & 1 & 7.85 & 19.50 & 1.25 \\
\hline 122 & 2.5 & 0 & 5 & 7.79 & 16.00 & 2.33 \\
\hline 104 & 0 & 2.5 & 2 & 7.79 & 15.67 & 1.25 \\
\hline 123 & 3 & 0 & 5 & 7.60 & 19.67 & 1.67 \\
\hline 116 & 0 & 2.5 & 1 & 7.58 & 18.00 & 1.33 \\
\hline 133 & 2 & 0 & 1 & 7.50 & 12.00 & 1.33 \\
\hline
\end{tabular}

Table 4. ROVER identified PIO incident summary (top 10) by axis: pitch (top), roll (bottom).

Biodynamic coupling is typically defined as an event in which the accelerations of an aircraft impact the stick input through vibrations of the pilot's arm. In a manner similar to PIOs, there exists a relationship between the accelerations of an aircraft and the stick input. However, there are distinguishing differences. The first is that these oscillations are no longer out of phase- instead fairly close to being in phase. The phase difference will be dependent upon the delays of the system and the frequency at which the oscillations are occurring. More simply put, the frequency dictates the period of a full oscillation and the phase shift is a portion of the period. Therefore, a delay between the acceleration being felt by the pilot and translated into a stick deflection and recorded as time series data, is then converted to a small phase shift where the stick lags the acceleration. Because the time delay of a system is typically time based fixed, the phase shift that is acceptable will be represented by a band because of the varying frequency possibilities. For the purpose of this study, the search for biodynamic coupling was limited to the DASE frequency spectrum. Another distinction is the stick deflection. In the case of a PIO, the stick input should be 
intentional as it is a product of the pilot trying to control the aircraft. So naturally, the threshold requires the stick input to be greater than a certain value. For biodynamic coupling, the input is not intentional and can therefore be a very small deflection change, or a large deflection change, depending on the amplitude of the accelerations felt and the pilot's grip on the stick as a form of bracing against the effects of the acceleration. The outputs of the ROVER biodynamic coupling routine are summarized in Table 5. Because the thresholds for peak-to-peak amplitudes of accelerations and stick input are far more sensitive in the biodynamic coupling case, the table also indicates the average number of frequency and phase shift matches. The engineering judgment required to classify an oscillation as biodynamic coupling beyond the score of 2 (F/P Match) is far greater in this case.

\begin{tabular}{|c|c|c|c|c|c|c|c|c|c|c|c|c|c|c|c|c|c|}
\hline \multicolumn{4}{|c|}{ Biodynamic Coupling- Pitch Accel } & \multicolumn{2}{|c|}{ All Pilots } & \multicolumn{2}{|c|}{ Pilot 1} & \multicolumn{2}{|c|}{ Pilot 2} & \multicolumn{2}{|c|}{ Pilot 3} & \multicolumn{2}{|c|}{ Pilot 4} & \multicolumn{2}{|c|}{ Pilot 5} & \multicolumn{2}{|c|}{ Pilot 6} \\
\hline Vehicle & A Freq $(\mathrm{Hz})$ & S Freq $(\mathrm{Hz})$ & Defl R & F/P Match & $\mathrm{BIOC}$ & F/P Match & $\mathrm{BIOC}$ & F/P Match & $\mathrm{BIOC}$ & F/P Match & $\mathrm{BIOC}$ & F/P Match & $\mathrm{BIOC}$ & F/P Match & $\mathrm{BIOC}$ & F/P Match & $\mathrm{BIOC}$ \\
\hline 109 & 0 & 2 & 1.5 & 3.95 & 0.35 & 5.67 & 0.00 & 6.00 & 0.33 & 3.67 & 0.00 & 4.00 & 0.25 & 1.25 & 0.50 & 4.00 & 1.00 \\
\hline 110 & 0 & 2.5 & 1.5 & 3.84 & 0.26 & 2.67 & 0.00 & 5.33 & 0.33 & 5.00 & 0.67 & 2.67 & 0.67 & 2.33 & 0.00 & 4.75 & 0.00 \\
\hline 116 & 0 & 2.5 & 1 & 3.95 & 0.26 & 0.33 & 0.00 & 5.67 & 0.00 & 5.50 & 0.25 & 4.00 & 0.00 & 3.00 & 0.33 & 4.67 & 1.00 \\
\hline 135 & 3 & 0 & 1 & 3.95 & 0.26 & 1.67 & 0.00 & 6.25 & 0.75 & 4.67 & 0.33 & 4.33 & 0.33 & 1.67 & 0.00 & 4.33 & 0.00 \\
\hline
\end{tabular}

\begin{tabular}{|c|c|c|c|c|c|c|c|c|c|c|c|c|c|c|c|c|c|}
\hline \multicolumn{4}{|c|}{ Biodynamic Coupling- Roll Accel } & \multicolumn{2}{|c|}{ All Pilots } & \multicolumn{2}{|c|}{\begin{tabular}{|l} 
Pilot 1 \\
\end{tabular}} & \multicolumn{2}{|c|}{ Pilot 2} & \multicolumn{2}{|c|}{ Pilot 3} & \multicolumn{2}{|c|}{ Pilot 4} & \multicolumn{2}{|c|}{ Pilot 5} & \multicolumn{2}{|c|}{ Pilot 6} \\
\hline Vehicle & A Freq $(\mathrm{Hz}$ & S Freq $(\mathrm{Hz})$ & Defl R & F/P Match & $\mathrm{BIOC}$ & F/P Match & $\mathrm{BIOC}$ & F/P Match & $\mathrm{BIOC}$ & F/P Match & $\mathrm{BIOC}$ & F/P Match & $\mathrm{BIOC}$ & F/P Match & $\mathrm{BIOC}$ & F/P Match & $\mathrm{BIOC}$ \\
\hline 114 & 0 & 1.5 & 1 & 18.42 & 3.65 & 15.43 & 8.14 & 22.67 & 0.33 & 13.75 & 1.25 & 24.00 & 2.67 & 19.33 & 2.83 & 20.00 & 2.33 \\
\hline 132 & 1.5 & 0 & 1 & 18.30 & 3.65 & 12.25 & 10.75 & 23.50 & 0.75 & 14.33 & 1.33 & 23.00 & 1.67 & 18.67 & 3.33 & 18.33 & 2.67 \\
\hline 104 & 0 & 2.5 & 2 & 18.26 & 3.37 & 14.00 & 7.67 & 23.50 & 0.25 & 12.33 & 1.33 & & & 22.33 & 1.67 & 21.67 & 2.67 \\
\hline 113 & 0 & 1 & 1 & 18.24 & 3.36 & 12.88 & 7.13 & 23.33 & 0.33 & 17.67 & 1.33 & 26.00 & 2.00 & 22.75 & 2.25 & 15.25 & 1.75 \\
\hline 135 & 3 & 0 & 1 & 18.16 & 3.00 & 12.00 & 9.67 & 23.75 & 0.00 & 15.33 & 0.33 & 19.00 & 1.67 & 22.00 & 4.33 & 15.00 & 3.00 \\
\hline 119 & 1 & 0 & 5 & 19.89 & 2.95 & 15.75 & 5.75 & 20.33 & 1.67 & 18.33 & 1.33 & 24.33 & 1.00 & 23.00 & 4.33 & 19.00 & 2.67 \\
\hline
\end{tabular}

\begin{tabular}{|c|c|c|c|c|c|c|c|c|c|c|c|c|c|c|c|c|c|}
\hline \multicolumn{4}{|c|}{ Biodynamic Coupling- Sway Accel } & \multicolumn{2}{|c|}{ All Pilots } & \multicolumn{2}{|c|}{ Pilot 1} & \multicolumn{2}{|c|}{ Pilot 2} & \multicolumn{2}{|c|}{ Pilot 3} & \multicolumn{2}{|c|}{ Pilot 4} & \multicolumn{2}{|c|}{ Pilot 5} & \multicolumn{2}{|l|}{ Pilot 6} \\
\hline Vehicle & A Freq $(\mathrm{Hz})$ & S Freq $(\mathrm{Hz})$ & Defl $R$ & F/P Match & $\mathrm{BIOC}$ & F/P Match & $\mathrm{BIOC}$ & F/P Match & $\mathrm{BIOC}$ & F/P Match & $\mathrm{BIOC}$ & F/P Match & $\mathrm{BIOC}$ & F/P Match & $\mathrm{BIOC}$ & F/P Match & $\mathrm{BIOC}$ \\
\hline 119 & 1 & 0 & 5 & 3.68 & 0.79 & 0.25 & 1.25 & 5.67 & 0.67 & 3.33 & 1.00 & 7.33 & 0.00 & 3.33 & 0.67 & 3.33 & 1.00 \\
\hline 125 & 1 & 0 & 3 & 3.80 & 0.45 & 1.67 & 0.67 & 6.33 & 0.33 & 2.33 & 1.00 & 5.00 & 0.25 & 4.00 & 0.67 & 3.25 & 0.00 \\
\hline 124 & 3.5 & 0 & 5 & 3.80 & 0.45 & 1.67 & 0.67 & 6.33 & 0.33 & 2.33 & 1.00 & 5.00 & 0.25 & 4.00 & 0.67 & 3.25 & 0.00 \\
\hline 118 & 0 & 3.5 & 1 & 12.56 & 0.33 & 12.00 & 1.33 & 15.33 & 0.00 & 11.00 & 0.00 & 15.33 & 0.00 & 9.33 & 0.67 & 12.33 & 0.00 \\
\hline 126 & 1.5 & 0 & 3 & 8.52 & 0.24 & 9.67 & 1.33 & 8.75 & 0.00 & 8.50 & 0.00 & 8.75 & 0.00 & 8.67 & 0.33 & 6.67 & 0.00 \\
\hline
\end{tabular}

Table 5. Biodynamic Coupling ROVER Output Summary: pitch acceleration top 3 (top), roll acceleration top 6 (middle) and sway acceleration top 5 (bottom).

It is necessary to point out a few key issues with automated software searches for these incidents of PIO and biodynamic coupling. With regards to PIOs, typically a frequency range of 1 to $9 \mathrm{rad} / \mathrm{sec}$ is used for selection of frequency. However, the DASE models added modes that are outside of the normal phugoid and short period modes. One of the goals of the study was to determine if there are increased likelihoods of PIO with added structural mode oscillations. Therefore the range of acceptable frequencies had to be opened up to $25 \mathrm{rad} / \mathrm{sec}$ to include the DASE frequencies. Unfortunately, this widened range opens up the search to include pilot-aircraft coupling incidents such as the previously mentioned pitch bobble and roll ratchet. Further aggravating this issue is that the average angular rates of the aircraft used in the study seemed to all occur below $10 \mathrm{deg} / \mathrm{sec}$. Therefore, the peak-topeak thresholds that would otherwise block out these bobbles and ratchets could not be instituted. Finally, the relationship between PIOs and biodynamic coupling is difficult to crisply define. In Fig. 9, ROVER detected a very strong PIO that persisted for about 25 seconds. Examining the spectral analysis of this run, it can be seen that the stick frequency was sitting right at the frequency of the DASE model- which confirms that the pilot was definitely coupled with the aircraft. Additionally, the pilot's post run comments indicate that he felt like he was experiencing biodynamic coupling. His average stick deflections were well below the peak-to-peak values seen here in his input. This typically indicates that to an extent, a majority of the input was intentional, likely indicating PIO. It is also worth saying that the overall accelerations on the vehicle, while definitely apparent, were below the threshold that ROVER would identify as viable to create a biodynamic coupling event.

Ultimately, while ROVER outputs reliable identification of frequency and phase flags, it often requires further manual analysis of the time series data and pilot comments to verify an actual occurrence. Even with all the information available, in cases similar to run 2420 in Fig. 9, an event may not be fully classifiable. In the end, the ROVER software with the new flag algorithm modifications provides timely, automated notifications of runs to evaluate further. Nonetheless, the results are promising and worthy of additional investigation and tuning of ROVER for this new biodynamic coupling application. 


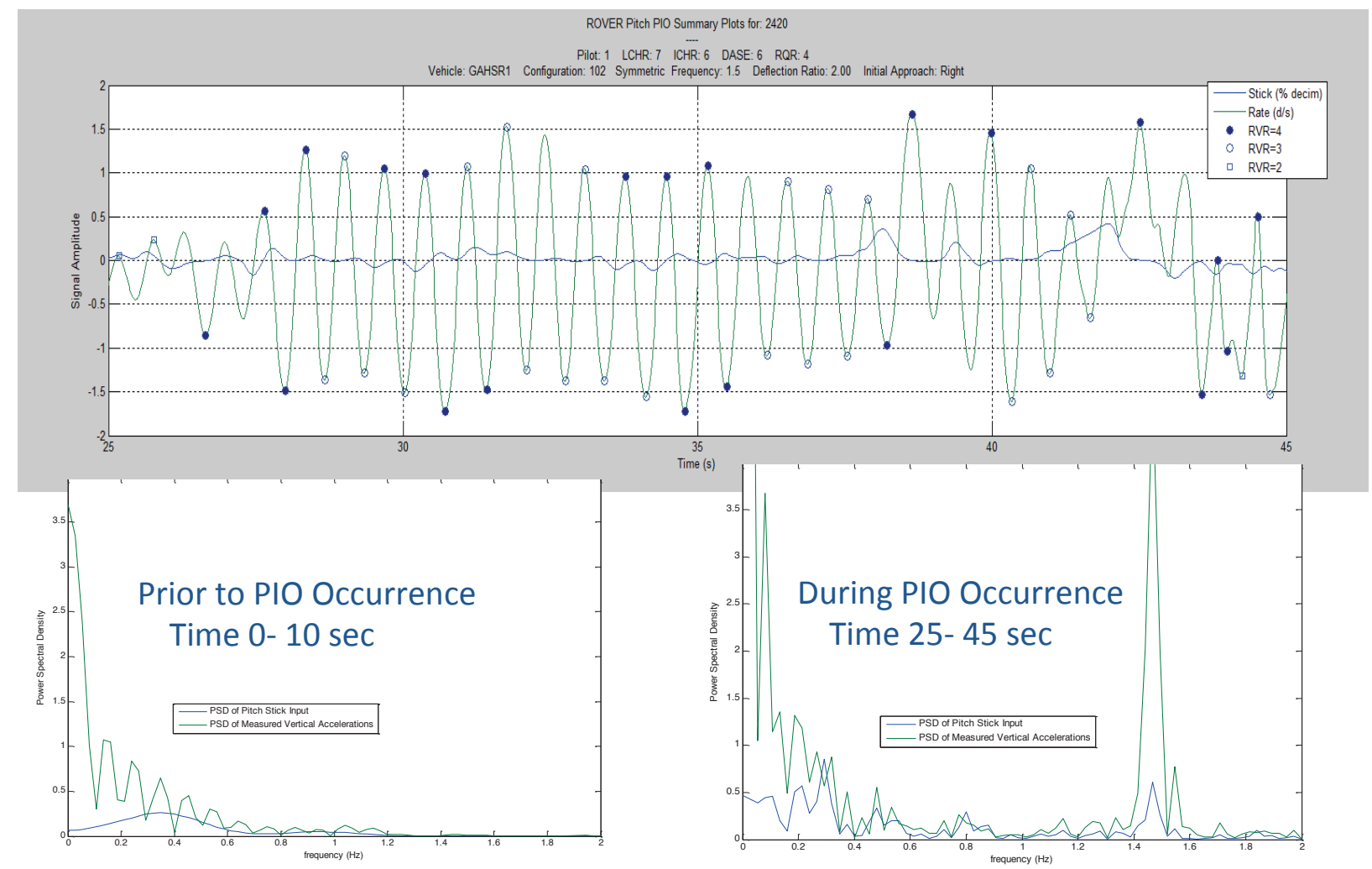

Figure 9. Example simulation run (2420) containing pitch PIO potentially aggravated by biodynamic coupling.

\section{Effects of DASE Models on Flight Technical Error}

As discussed in the earlier section regarding control strategy, flight technical error showed little correlation to particular vehicles, axis, or even frequency. The average of the deviations by vehicle, which were calculated using absolute values of the errors, from the overall mean performance of all runs, was unsubstantial. In Table 6, it is seen that vehicles 113 and 119, both associated with a DASE frequency of $1 \mathrm{~Hz}$ (though different axes) tended to drive up some of the maximum errors. The table provides the top three maximum error incidents by vehicle for the highlighted field. This trend did not translate to the average errors by vehicle- it was isolated only to maximum incidents. This $1 \mathrm{~Hz}$ modal frequency consistently had maximum errors that were in the top three of all vehicles and were considerably outside the adequate performance category summarized in Table 1. Similar trends persisted for the top three vehicle errors sorted in the other performance categories (other table columns), but these have not been included for brevity.

\begin{tabular}{|c|c|c|c|c|c|c|c|c|c|c|c|}
\hline Vehicle & AS Frea & Sym Freg & Refl Ratio & AS $50 \mathrm{ft}$ Frr & Bank It 50ft Frr & I & GSDev $300 \mathrm{ft}$ Frr & & & $\mathrm{Y} M \mathrm{MG}$ TR Err & \\
\hline 113 & 0 & 1 & - mon- & & & & & & & & \\
\hline & & & & & & & & & & & \\
\hline 119 & 1 & 0 & 5 & 2.400 & 29.580 & 3.150 & 1.970 & 2.400 & 9.190 & 849.980 & 69.410 \\
\hline 118 & 0 & 3.5 & 1 & 1.560 & 22.240 & 2.420 & 1.250 & 2.990 & 7.250 & 823.860 & 37.280 \\
\hline
\end{tabular}

\begin{tabular}{|c|c|c|c|c|c|c|c|c|c|c|c|}
\hline Vehicle & AS Freg & Sym Freq & Defl Ratio & AS 50ft Err & Bank It $50 \mathrm{ft}$ Err & LocDev $300 \mathrm{ft}$ Err & GSDev $300 \mathrm{ft}$ Err & Ldg Hdg Err & Ldg Hdot Err & $\mathrm{X}$ MG TD Err & Y MG TD Err \\
\hline 113 & 0 & 1 & 1 & 2.470 & 33.310 & 4.220 & 0.790 & 3.220 & 10.550 & 907.180 & 12.550 \\
\hline 119 & 1 & 0 & 5 & 2.400 & 29.580 & 3.150 & 1.970 & 2.400 & 9.190 & 849.980 & 69.410 \\
\hline 104 & 0 & 2.5 & 2 & 1.680 & 28.470 & 1.080 & 0.620 & 2.020 & 13.250 & 796.820 & 18.700 \\
\hline
\end{tabular}

ALL PILOTS Max Errors by Vehicle

\begin{tabular}{|c|c|c|c|c|c|c|c|c|c|c|c|}
\hline Vehicle & AS Freq & Sym Freq & Defl Ratio & AS_50ft_Err & Bank_It_50ft_Err & LocDev_300ft_Err & GSDev_300ft_Err & Ldg_Hdg_Err & Ldg_Hdot_Err & X_MG_TD_Err & Y_MG_TD_Err \\
\hline 115 & 0 & 2 & 1 & 1.920 & 13.690 & 0.940 & 2.510 & 3.820 & 12.450 & 1149.940 & 46.340 \\
\hline 119 & 1 & 0 & 5 & 2.400 & 29.580 & 3.150 & 1.970 & 2.400 & 9.190 & 849.980 & 69.410 \\
\hline 126 & 1.5 & 0 & 3 & 2.130 & 15.410 & 0.960 & 1.780 & 2.940 & 7.500 & 950.400 & 24.610 \\
\hline
\end{tabular}

Table 6. Maximum Flight Technical Error Occurrences - Top 3: localizer deviation error at 300ft (top), maximum bank angle less than 50ft (middle) and glideslope deviation error at 300ft (bottom).

(See Table 1 for definition of parameter names) 
Even less significant is the correlation between the ratings and the average flight technical error for the runs receiving that rating. As Table 7 summarizes, there is very little variation in the performance between Level 2 and Level 3 Cooper Harper ratings in both the landing and ILS tracking portions of the task. It should be pointed out that there are cases when the average error was less for the Level 3 runs than for the Level 2 runs. It can be speculated that the pilot was more engaged during these runs, and though his workload to maintain control through the additional DASE incited vibrations, his focus was more specifically directed to meeting the adequate or desired performance levels as-briefed. The column titles in Table 6 and 7 correspond to the performance metrics outlined in Table 1. The elements of the Table 7 are calculated as the averages of the absolute value of the errors per run that received a rating associated with the row header.

\begin{tabular}{|c|c|c|c|c|c|c|c|c|}
\hline AVERAGES: & AS_50ft_Err & Bank_It_50ft_Err & LocDev_300ft & GSDev_300ft_Err & Ldg_Hdg_Err & Ldg_Hdot_Error & X_MG_TD_Err & Y_MG_TD_Error \\
\hline ICHR LvI 2 & 1.064 & 3.903 & 0.365 & 0.315 & 1.241 & 3.891 & 358.513 & 9.021 \\
\hline ICHR LVI 3 & 0.641 & 3.195 & 0.446 & 0.443 & 0.555 & 2.633 & 579.325 & 17.018 \\
\hline LCHR Lvl 2 & 1.042 & 3.960 & 0.323 & 0.302 & 1.148 & 4.066 & 312.216 & 8.351 \\
\hline LCHR Lvl 3 & 0.872 & 5.657 & 0.326 & 0.330 & 0.830 & 4.090 & 319.363 & 9.127 \\
\hline DASE 123 & 1.045 & 3.921 & 0.313 & 0.288 & 1.104 & 4.032 & 319.761 & 8.210 \\
\hline DASE 456 & 0.806 & 4.846 & 0.286 & 0.307 & 0.775 & 3.744 & 265.100 & 7.347 \\
\hline RQR 456 & 1.004 & 4.286 & 0.330 & 0.316 & 1.136 & 3.729 & 335.467 & 8.533 \\
\hline
\end{tabular}

Table 7. Flight Technical Error (average) by ratings.

(See Table 1 for definition of parameter names)

\section{E. Facility Considerations}

The CMF characteristics are in the early phases of being quantified. It was discovered during the course of this study that the hydraulic system in its current state exhibits parasitic, or cross-axis, coupling in uncommanded axes. Steps were taken to mitigate this anomaly prior to the study. The cross-coupling was noticeably occurring when the motion platform was off-center and with certain combinations of commands. This type of cross coupling is a standard occurrence for this type of mechanical hydraulically actuated system due to its highly nonlinear nature. Output position plots for pure back and forth motions show more of an elliptical behavior. These plots will be available in future reports that intend to illustrate the performance of the Langley CMF simulator. At this time, it is unknown how often and to what degree that this cross-coupling affected the data and pilot assessments of the particular DASE models. In the effort to quantify the cross-coupling, it was also noted that the simulator exhibited harmonic excitation in numerous circumstances. The cross-coupling has been seen to occur not only in the DASE frequency, but also at DASE frequency harmonics, in both the DASE axis and two other non-DASE axes. Because the study focused on single axis DASE models, it is expected that the instances of cross-coupling should be identifiable and quantifiable. As far as the authors are aware, this approach is unique. It increases demands on simulation motion systems beyond what it typically seen.

Given that the results show that effects of the $3.5 \mathrm{~Hz}$ DASE models are not nearly as significant as the lower frequencies, the concerns regarding the limitations of the motion simulator's $3 \mathrm{~Hz}$ production control law appear to have been mitigated for the current study. Further analysis of the typical phase delays associated with this higher frequency will be conducted and published. No significant cross-coupling has been identified at this frequency for this study.

\section{Concluding Remarks}

Based on the initial findings of this study, a number of structural model frequency and deflection ratio combinations have been identified as objectionable in terms of aircraft handling qualities deterioration. Further analysis of the data is ongoing and will be documented in a NASA Technical Paper. This analysis includes, but is not limited to, the effects of the structural characteristics on the pilots' control strategy, the relationship between pilot physical build and biodynamic coupling, the contribution of PIOs to biodynamic coupling, and the effects of simulator cross-coupling on the data. The objective of this work is to provide guidance to supersonic civilian transport design teams related to the handling qualities degradation and biodynamic coupling associated with these specific rigid body dynamics and elastic mode pairings. The study results will be useful for defining structural design limitations and control augmentation system requirements for supersonic civilian transport studies. 


\section{Acknowledgments}

The authors would like to acknowledge Gustav Taylor (UniSYS) and Victoria Chung of the Simulation Development and Analysis Branch at NASA Langley for their help and cooperation with the CMF, as well as Melissa Proffitt of AAG for her assistance in data collection and pilot comment transcriptions. 


\section{References}

${ }^{1}$ Raney, David L., Jackson, E. Bruce, and Butrill, Carey S., "Simulation Study of Impact of Aeroelastic Characteristics on Flying Qualities of a High Speed Civil Transport,” NASA/TP-2002-211943, 2002.

${ }^{2}$ Jackson, E. Bruce, Martinez, Debbie, and Derry, Stephen D., "Piloted Simulation Investigation of Supersonic Transport Configuration (LaRC.4)," NASA/TM-1999-209557, 1999.

${ }^{3}$ Harper, R P, Jr. and Cooper, G E., "Handling Qualities and Pilot Evaluation," AIAA Journal of Guidance, Control, and Dynamics, Vol. 9, Sept.-Oct. 1986, pp. 515-529.

${ }^{4}$ Anon., "Military Standard, Flying Qualities of Piloted Vehicles,” MIL-STD-1798A, January 30, 1990.

${ }^{5}$ Hoffler, Keith D., Fears, Scott P., and Carzoo, Susan W., "Generic Airplane Model Concept and Four Specific Models Developed for Use in Piloted Simulation Studies,” NASA CR 201651, 1997.

${ }^{6}$ Stringer, Mary T., Cowen, Brandon, and Hoffler, Keith D., "Scalable Prototyping Aircraft for Rapidly Configured 6DOF Models: SPARC6," NASA/CR-2013 (to be published).

${ }^{7} J a c k s o n$, E. Bruce. and Proffitt, Melissa, "HOPE: An On-Line Piloted Handling Qualities Experiment Data Book,” AIAA [1] Atmospheric Flight Mechanics Conference, Toronto, Canada, 2010.

${ }^{8}$ MATLAB version 2013a. Natick, Massachusetts: The MathWorks Inc., 2013.

${ }^{9}$ Mitchell, David G., and Klyde, David H., "Identifying a Pilot-Induced Oscillation Signature: New Techniques Applied to Old Problems," Journal of Guidance, Control and Dynamics, Vol. 3, No. 1, Jan.-Feb. 2008. 